Research Article

Open Access

Special Issue: Recent Developments in Quantitative Risk Management

Giovanni Puccetti`, Ludger Rüschendorf, and Dennis Manko

\title{
VaR bounds for joint portfolios with dependence constraints
}

DOI 10.1515/demo-2016-0021

Received June 14, 2016; accepted October 4, 2016

\begin{abstract}
Based on a novel extension of classical Hoeffding-Fréchet bounds, we provide an upper VaR bound for joint risk portfolios with fixed marginal distributions and positive dependence information. The positive dependence information can be assumed to hold in the tails, in some central part, or on a general subset of the domain of the distribution function of a risk portfolio. The newly provided VaR bound can be interpreted as a comonotonic VaR computed at a distorted confidence level and its quality is illustrated in a series of examples of practical interest.
\end{abstract}

Keywords: Value-at-Risk; Dependence Uncertainty; Positive Dependence; Model Risk

MSC: 91B30 (primary); 60E15 (secondary)

\section{Motivation and preliminaries}

Establishing reliable bounds for the Value-at-Risk (VaR) of a joint portfolio is a relevant subject in connection with the amount of risk capital in the Basel II/III regulations for the finance sector as well as with the Solvency regulations for the insurance sector. A series of results and different methods have been established in the last decade to find good bounds for the VaR of a joint portfolio based on available information on its dependence structure.

The paper [15] gives a survey of the methods available concerning the computation of VaR bounds when only the marginal distributions of the individual risk factors of a portfolio are known.

For the case where extra dependence information is assumed, improved VaR bounds are also available in the literature. For instance, in [14, Section 4] it is shown that higher order (typically bi-dimensional) marginal information on the joint portfolio, when available, may lead to strongly improved bounds. The worst VaR bound can be similarly reduced by estimating the values of the copula on some subset of its domain (see [2]) or putting a variance constraint on the total position (see [3]). Effects of this dependence information on the reduction of the VaR bounds are described in [6] and in [2]. Some higher order marginal information has been investigated in [13], [23], [14], and in [26]. The reduction of VaR bounds by inclusion of additional second or higher order moment information was described in [3] and in [4].

This paper continues the streamline of easily computable and practical bounds on VaR which has been initiated in [6] and further extended in [26]. Based on a novel extension of classical Hoeffding-Fréchet bounds, we provide an upper VaR bound for a joint risk portfolio with fixed marginal distributions and positive dependence information assumed on a subset of the domain of its distribution function. The newly provided VaR bound can be interpreted as a comonotonic VaR computed at a distorted confidence level and its quality is illustrated in a series of examples.

`Corresponding Author: Giovanni Puccetti: Department of Economics, Management and Quantitative Methods, University of Milano, Italy

Ludger Rüschendorf, Dennis Manko: Department of Mathematical Stochastics, University of Freiburg, Germany 


\section{Summary of the paper}

In Section 2, we set the probabilistic framework and study so-called improved standard bounds on the VaR of a joint portfolio. These bounds are not new but we provide some insight on their computation which will turn out to be useful in the remainder of the paper.

In Section 3 we state an extension of classical Hoeffding-Fréchet bounds and we use this extension to weaken the assumptions under which the improved VaR bounds given in Section 2 hold. In particular we consider the case when positive dependence information is assumed to hold in the tails, in some central part, or on a general subset of the domain of the distribution function of a risk portfolio. We provide several applications and numerical results which show that the improvement given by the novel bounds can be considerable depending on the dependence information provided.

\section{Value-at-Risk.}

Throughout the paper, the quasi-inverse $f^{-1}$ of an increasing function $f: A \subset \mathbb{R} \rightarrow[0,1]$ is defined as

$$
f^{-1}(u):=\inf \{x \in A: f(x) \geq u\}, \text { for } u \in(0,1],
$$

and $f^{-1}(0):=\inf \{x \in A: f(x)>0\}$. The Value-at-Risk (VaR) of a loss random variable $Y$, computed at a probability level $\alpha \in(0,1)$, is then defined as

$$
\operatorname{VaR}_{\alpha}(Y)=F_{Y}^{-1}(\alpha)=\inf \left\{x \in \mathbb{R}: F_{Y}(x) \geq \alpha\right\},
$$

where $F_{Y}(x)=P(Y \leq x)$ is the distribution function of $Y$.

\section{Basic notions of copula theory.}

Throughout the paper we extensively use the concept of a copula to model the dependence structure within a risk portfolio. The reader not familiar with the theory of copulas is referred to the textbook references [21], [19] and [9].

A copula $C$ is a $n$-dimensional distribution function on $[0,1]^{n}$ with uniform marginals. Given a copula $C$ and $n$ univariate marginals $F_{1}, \ldots, F_{n}$, one can always define a distribution $F$ on $\mathbb{R}^{n}$ having these marginals by

$$
F\left(x_{1}, \ldots, x_{n}\right)=C\left(F_{1}\left(x_{1}\right), \ldots, F_{n}\left(x_{n}\right)\right), x_{1}, \ldots, x_{n} \in \mathbb{R} .
$$

Sklar's theorem states conversely that we can always find a copula $C$ coupling the marginal distributions of a fixed joint distribution $F$ through the above expression (1.1). For continuous marginal distributions, this copula is unique. A basic example of a copula is the independence copula $\Pi\left(u_{1}, \ldots, u_{n}\right)=\Pi_{i=1}^{n} u_{i}$. The name of this copula derives from the fact that the risk vector $\left(X_{1}, \ldots, X_{n}\right)$ has copula $\Pi$ if and only if its marginal risks $X_{i}$ are independent.

It is well known that the joint distribution $F_{X}$ of a random vector $X$ with marginal distributions $F_{1}, \ldots, F_{n}$ satisfies

$$
\underline{F}(x) \leq F_{X}(x) \leq \bar{F}(x), x \in \mathbb{R}^{n},
$$

where

$$
\underline{F}(x)=\left(\sum_{i=1}^{n} F_{i}(x)-n+1\right)_{+} \quad \text { and } \quad \bar{F}(x)=\min _{1 \leq i \leq n} F_{i}\left(x_{i}\right)
$$

are the so-called lower and, respectively, upper Hoeffding-Fréchet bounds. Setting $F_{i}=U(0,1), 1 \leq i \leq n$, one obtains the corresponding bounds for any copula $C$ :

$$
W(u) \leq C(u) \leq M(u), u \in[0,1]^{n},
$$

where $W(u)=\left(u_{1}+\cdots+u_{n}-n+1\right)_{+}$and $M(u)=\min \left\{u_{1}, \ldots, u_{n}\right\}$. The upper Fréchet bound $M$ is also called the comonotonic copula and represents perfect positive dependence among the marginal risks. The lower Fréchet 
bound $W$ is a well-defined copula only in dimension $n=2$. In this case, it is called the countermonotonic copula and represents perfect negative dependence between two risks.

When the copula of a risk vector $X=\left(X_{1}, \ldots, X_{n}\right)$ is equal to $M$, that is when the marginal risks $X_{i}$ are assumed to be comonotonic, we have that the VaR for the aggregate portfolio $S=\sum_{i=1}^{n} X_{i}$ is given by

$$
\operatorname{VaR}(S)=\operatorname{VaR}_{\alpha}^{+}(S),
$$

where $\operatorname{VaR}_{\alpha}^{+}(S)$ is the so-called comonotonic VaR defined as the VaR of the comonotonic sum and given by

$$
\operatorname{VaR}_{\alpha}^{+}(S)=\sum_{i=1}^{n} \operatorname{VaR}_{\alpha}\left(X_{i}\right)=\sum_{i=1}^{n} F_{i}^{-1}(\alpha) .
$$

In order to model some dependence scenarios, in the remainder of the paper we will also use the following copula families:

(1) The Gumbel copula with parameter $\theta \geq 1$,

$$
C_{\theta}^{G u}\left(u_{1}, \ldots, u_{n}\right)=\exp \left(-\left[\left(-\ln u_{1}\right)^{\theta}+\cdots+\left(-\ln u_{n}\right)^{\theta}\right]^{1 / \theta}\right),
$$

The Gumbel copula interpolates between independence $\left(C_{1}^{G u}=\Pi\right)$ and comonotonic dependence $\left(C_{\theta \rightarrow \infty}^{G u}=M\right)$. The parameter $\theta$ can easily be calibrated using Kendall's tau $\tau=1-\theta^{-1}$ and exhibits upper tail dependence $\lambda_{u}\left(C_{\theta}^{G u}\right)=2-2^{1 / \theta}$.

(2) The Gaussian copula with correlation parameter $-1 \leq \rho \leq 1$,

$$
C_{\rho}^{G a}\left(u_{1}, \ldots, u_{n}\right)=\Phi_{\rho}\left(\Phi^{-1}\left(u_{1}\right), \ldots, \Phi^{-1}\left(u_{n}\right)\right) .
$$

Here $\Phi_{\rho}$ denotes the joint distribution of a zero-mean Gaussian random vector with equicorrelation matrix $\Sigma$, with ones on the main diagonal and off-diagonal elements equal to $\rho . \Phi^{-1}$ is the quantile function of a standard normal random variable. Similarly to the Gumbel, the Gaussian copula interpolates for $0 \leq \rho \leq 1$ between independence $\left(C_{0}^{G a}=\Pi\right)$ and the comonotonic limiting case $\left(C_{1}^{G a}=M\right)$, and may as well capture negative dependence when $\rho<0$. In contrast however to $C_{\theta}^{G u}, C_{\rho}^{G a}$ for $\rho<1$ is asymptotically tail independent, $\lambda_{u}\left(C_{\rho}^{G a}\right)=0$. The Kendall's tau rank correlation parameter is equal to $\tau=\frac{2}{\pi} \arcsin \rho$.

\section{Improved VaR bounds with dependence information}

For a risk vector $X=\left(X_{1}, \ldots, X_{n}\right)$ with $X_{i} \sim F_{i}, 1 \leq i \leq n$, it is a classical problem to find good (best possible) bounds for the distribution function or, equivalently, for the Value-at-Risk of the joint portfolio $S=\sum_{i=1}^{n} X_{i}$. The worst-possible VaR value for $S$ consistent with the fixed marginals is defined by

$$
\overline{\operatorname{VaR}}_{\alpha}(S)=\sup \left\{\operatorname{VaR}_{\alpha}\left(X_{1}+\cdots+X_{n}\right): X_{i} \sim F_{i}, 1 \leq i \leq n\right\} .
$$

To determine the exact value of $\overline{\operatorname{VaR}}_{\alpha}(S)$ there exist exact primal and dual representations which are difficult to evaluate or not available in general, see [25] and [35]. For general risk vectors, $\overline{\mathrm{VaR}}_{\alpha}(S)$ can be numerically evaluated via the Rearrangement Algorithm (RA) as described in [14]. It is well known (see for instance [15] and references therein) that $\overline{\operatorname{VaR}}_{\alpha}(S)$ can be much larger than the comonotonic VaR defined in (1.3).

In order to moderate the worst-possible VaR estimate, we assume that positive dependence information is available. Let $C_{l}:[0,1]^{n} \rightarrow[0,1]$ be a componentwise increasing function satisfying

$$
W(u) \leq C_{l}(u) \leq M(u), u \in[0,1]^{n} .
$$

Positive dependence will be introduced in the form that $a$ copula $C_{X}$ of the risk vector $X$ satisfies $C_{X}(u) \geq$ $C_{l}(u), u \in[0,1]^{n}$. When the marginals of a risk vector are fixed and $C_{l}$ is a copula, this corresponds to the 
assumption $X \geq_{\text {lo }} Y$, namely $X$ is larger in the lower orthant order than $Y$, where $Y$ is a risk vector with the same marginal distributions of $X$ and copula $C_{l}$. For more details on the lower orthant order, we refer the reader to [31]. However, we stress that, for the results stated in this paper, $C_{l}$ does not need to be a copula; see Remark 3.2(2.) in [23]. For instance, $C_{l}$ can be taken as a quasi-copula.

The following bound on the distribution of a joint portfolio with given marginals and lower bound on its copula has appeared in various forms in the literature; see for instance Theorems 3.1 and 4.1 in [10] and Theorem 3.11 in [30].

Proposition 2.1 (Improved standard bound). Let $\left(X_{1}, \ldots, X_{n}\right)$ be a risk vector having marginal distribution functions $F_{1}, \ldots, F_{n}$ and copula $C_{X}$ satisfying $C_{X} \geq C_{l}$. Then

$$
P(S \leq t) \geq \tau_{C_{l}}(t):=\sup _{x \in \mathcal{U}(t)} C_{l}\left(F_{1}\left(x_{1}\right), \ldots, F_{n}\left(x_{n}\right)\right)
$$

where $U(t)=\left\{x=\left(x_{1}, \ldots, x_{n}\right) \in \mathbb{R}^{n}: \sum_{i=1}^{n} x_{i}=t\right\}$. The previous inequality is equivalent to the following VaR bound:

$$
\operatorname{VaR}_{\alpha}(S) \leq \tau_{C_{l}}^{-1}(\alpha)=\inf _{u \in \mathcal{Z}(\alpha)} \sum_{i=1}^{n} F_{i}^{-1}\left(u_{i}\right),
$$

where $z(\alpha)=\left\{u=\left(u_{1}, \ldots, u_{n}\right) \in[0,1]^{n}: C_{l}\left(u_{1}, \ldots, u_{n}\right)=\alpha\right\}$.

\section{Remark 1.}

1) When $C_{l}=W$, no dependence information is assumed on top of the marginals and (2.1) gives the so-called standard bound which has been derived in several ways in the literature; a history of the problem is given in Example 4.1 in [27]. Hence the terminology of improved standard bound is adopted in Proposition 2.1. The standard bound $\tau_{W}(t)$ is in general sharp only when $n=2$; for counterexamples when $n \geq 3$ see [12]. However, under some restrictive assumptions $\tau_{W}(t)$ is sharp also in higher dimensions $n \geq 3$, e.g. when $W$ is a distribution function (see [7]).

Note however that in higher dimensions the Rearrangement Algorithm ([14]) allows to compute the sharp bound $\overline{\operatorname{VaR}}_{\alpha}(S)$ for risk vectors with general marginal distributions.

2) When $C_{X} \geq C_{l}>W$, dependence information within the risk vector $X$ is introduced and we will show that, even if in general $\tau_{C_{l}}(t)$ is sharp only for $n=2$, the improvement given by (2.2) compared to $\overline{\operatorname{VaR}}_{\alpha}(S)$ can be considerable.

3) Similar inequalities also hold for monotone increasing aggregation functions $\psi(X)$ replacing the sum $S=$ $\sum_{i=1}^{n} X_{i}$; see [10], [30], [23] and references therein.

\subsection{Explicit computation of improved standard bounds}

The improved standard bound in (2.1) is not easy to determine in general in explicit form. Proposition 3.2 in [20] gives an easily computable expression of (2.1) when one assumes that the marginal distributions are identical with ultimately decreasing density and the copula bound $C_{l}$ is symmetric and satisfies an extra second-order condition. In the following theorem we give a set of assumptions based on the notion of Schurconcavity which are simple to check. For a definition and more details about Schur-concavity we refer the reader to Chapter 3 in [18]. The diagonal section of a copula $C$ is the function $\delta_{C}:[0,1] \rightarrow[0,1]$ defined as $\delta_{C}(u)=C(u, \ldots, u)$. The definition holds similarly for general increasing functions $C_{l}$.

Theorem 2.2. Let $\left(X_{1}, \ldots, X_{n}\right)$ be a risk vector having identical marginal distribution functions $F_{1}=\cdots=$ $F_{n}=F$ and copula $C_{X}$ satisfying $C_{X} \geq C_{l}$. Further assume that:

(i) $C_{l}$ is Schur-concave;

(ii) $F(x)$ is concave for all $x \geq F^{-1}(\alpha)-\xi$, for some $\xi>0$. 
Then, we have that

$$
\tau_{C_{l}}^{-1}(\alpha)=n F^{-1}\left(\alpha^{\star}\right)
$$

where $\alpha^{\star}=\delta_{C_{l}}^{-1}(\alpha)$

Proof. From the definition of a quasi-inverse we have that

$$
\begin{aligned}
\tau_{C_{l}}^{-1}(\alpha) & =\inf \left\{t \in \mathbb{R}: \tau_{C_{l}}(t) \geq \alpha\right\} \\
& =\inf \left\{t \in \mathbb{R}: \sup _{\sum_{i=1}^{n} x_{i}=t} C_{l}\left(F\left(x_{1}\right), \ldots, F\left(x_{n}\right)\right) \geq \alpha\right\} .
\end{aligned}
$$

Note that when $x_{i} \rightarrow F^{-1}(\alpha)-\xi$ we have $C_{l}(\cdot)<\alpha$. Thus, we can restrict to considering $x_{i} \geq F^{-1}(\alpha)-\xi$ and rewrite

$$
\tau_{C_{l}}^{-1}(\alpha)=\inf \left\{t \geq n\left(F^{-1}(\alpha)-\xi\right): \sup _{\substack{\sum_{i=1}^{n} x_{i}=t, x_{i} \geq F^{-1}(\alpha)-\xi, 1 \leq i \leq n}} C_{l}\left(F\left(x_{1}\right), \ldots, F\left(x_{n}\right)\right) \geq \alpha\right\} .
$$

Since $F\left(x_{i}\right)$ is concave for $x_{i} \geq F^{-1}(\alpha)-\xi$ and $C_{l}$ is Schur-concave by assumption, from point (ii) in Table 2 (p. 91) in [18] we obtain that $C_{l}\left(F\left(x_{1}\right), \ldots, F\left(x_{n}\right)\right)$ is Schur-concave and the supremum in (2.4) is attained at the point $(t / n, \ldots, t / n)$, i.e.

$$
\sup _{\substack{\sum_{i=1}^{n} x_{i}=t, x_{i} \geq F^{-1}(\alpha)-\xi \\ 1 \leq i \leq n}} C_{l}\left(F\left(x_{1}\right), \ldots, F\left(x_{n}\right)\right)=C_{l}(F(t / n), \ldots, F(t / n))=\delta_{C_{l}}(F(t / n)) .
$$

Let $\alpha^{\star}=\delta_{C_{l}}^{-1}(\alpha)$. Since $W \leq C_{l} \leq M$, notice that $\delta_{C_{l}}(u) \leq u, u \in[0,1]$, and therefore $\alpha^{\star} \geq \alpha$. Then, we finally obtain that

$$
\begin{aligned}
\tau_{C_{l}}^{-1}(\alpha) & =\inf \left\{t \geq n\left(F^{-1}(\alpha)-\xi\right): \delta_{C_{l}}(F(t / n)) \geq \alpha\right\} \\
& =\inf \left\{t \geq n\left(F^{-1}(\alpha)-\xi\right): F(t / n) \geq \alpha^{\star}\right\}=n F^{-1}\left(\alpha^{\star}\right) .
\end{aligned}
$$

\section{Remark 2.}

1) Every Schur-concave copula is symmetric, but the converse implication is not true. The class of Schurconcave copulas is relevant in practice as for instance it includes all Archimedean (hence also the Gumbel) copulas; see [8] for the corresponding result and more details on the notion of Schur-concavity.

2) For marginal distributions $F$ of class $C_{2}$, assumption (ii) in Theorem (2.2) holds whenever $F$ has a decreasing density for all $x \geq F^{-1}(\alpha)-\xi$.

3) Also in several cases of non-symmetric bounding functions $C_{l}$ and of inhomogeneous marginals some results on exact solutions of (2.2) can be stated. The numerical evaluation of these solutions is typically not easy; for some examples see [11].

We note that a very easily computable VaR bound follows from (2.2) by choosing the vector $\left(\alpha^{\star}, \ldots, \alpha^{\star}\right) \in$ $z(\alpha)$. Since the diagonal section of $C_{l}$ is continuous we have that $\delta_{C_{l}}\left(\alpha^{\star}\right)=C_{l}\left(\alpha^{\star}, \ldots, \alpha^{\star}\right)=\alpha$. This implies the following VaR bound which holds in full generality for any feasible $C_{l}$ and any marginal distributions $F_{i}$.

Theorem 2.3. Assume that the risk vector $\left(X_{1}, \ldots, X_{n}\right)$ has fixed marginal distributions $F_{1}, \ldots, F_{n}$ and copula $C_{X} \geq C_{l}$. Then

$$
\operatorname{VaR}_{\alpha}(S) \leq \operatorname{VaR}_{\alpha^{\star}}^{+}(S)=\sum_{i=1}^{n} F_{i}^{-1}\left(\alpha^{\star}\right)
$$

where $\alpha^{\star}=\delta_{C_{l}}^{-1}(\alpha)$. 
The general VaR bound $\mathrm{VaR}_{\alpha^{*}}^{+}(S)$ given in Theorem 2.3 is just the comonotonic VaR for the joint portfolio $S$, computed at a distorted confidence level $\alpha^{\star}$. This bound is straightforward to implement for all marginal distributions and copula families commonly used in quantitative risk management. For instance, when $\delta_{C_{l}}$ is also strictly increasing, $\alpha^{\star}$ can be easily computed as the unique point such that $\delta_{C_{l}}\left(\alpha^{\star}\right)=\alpha$.

Recall from the proof of Theorem 2.2 that $\alpha^{\star} \geq \alpha$ and therefore that

$$
\operatorname{VaR}_{\alpha^{*}}^{+}(S) \geq \operatorname{VaR}_{\alpha}^{+}(S) \text {. }
$$

When $C_{X} \geq C_{l}=M$, i.e. $C_{X}=M$, we obviously have $\alpha^{\star}=\alpha$ and $\operatorname{VaR}_{\alpha^{\star}}^{+}(S)=\operatorname{VaR}_{\alpha}^{+}(S)$.

\section{Improved Hoeffding-Fréchet bounds under dependence restrictions}

Our first aim in this section is to obtain improved Hoeffding-Fréchet bounds (see (1.2)) for the distribution of $X$ under an extra dependence assumption. These improved bounds will then be used to weaken the assumptions under which Theorem 2.3 holds. Let $G: \mathbb{R}^{n} \rightarrow \mathbb{R}$ be an increasing function such that

$$
\underline{F}(x) \leq G(x) \leq \bar{F}(x), x \in \mathbb{R}^{n} .
$$

We assume that, for a distribution $F$ with fixed marginals $F_{1}, \ldots, F_{n}$, it is known that $F \leq G$ and/or that $F \geq G$ on some subset $S \subset \mathbb{R}^{n}$.

Theorem 3.1 (Improved Hoeffding-Fréchet bounds).

Let $S \subset \mathbb{R}^{n}$ and let $G: \mathbb{R}^{n} \rightarrow \mathbb{R}$ be an increasing function satisfying (3.1). Define the improved upper and lower Hoeffding-Fréchet bounds $F^{\star}$ and $F \star$ as:

$$
\begin{aligned}
& F^{\star}(x)=\min \left(\min _{1 \leq i \leq n} F_{i}\left(x_{i}\right), \inf _{y \in S}\left\{G(y)+\sum_{i=1}^{n}\left(F_{i}\left(x_{i}\right)-F_{i}\left(y_{i}\right)\right)_{+}\right\}\right), \\
& F_{\star}(x)=\max \left(0, \sum_{i=1}^{n} F_{i}\left(x_{i}\right)-(n-1), \sup _{y \in S}\left\{G(y)-\sum_{i=1}^{n}\left(F_{i}\left(y_{i}\right)-F_{i}\left(x_{i}\right)\right)_{+}\right\}\right) .
\end{aligned}
$$

For a n-variate distribution function $F$ with marginals $F_{1}, \ldots, F_{n}$, it holds that:

(i) If $F(y) \leq G(y)$ for all $y \in S$, then $F(x) \leq F^{\star}(x)$, for all $x \in \mathbb{R}^{n}$.

(ii) If $F(y) \geq G(y)$ for all $y \in S$, then $F(x) \geq F_{*}(x)$, for all $x \in \mathbb{R}^{n}$.

(iii) If $F(y)=G(y)$ for all $y \in S$, then $F_{\star}(x) \leq F(x) \leq F^{\star}(x)$ for all $x \in \mathbb{R}^{n}$.

Proof. (i) Let $X=\left(X_{1}, \ldots, X_{n}\right)$ be a random vector with distribution $F$ and w.l.g. let $y \in S$ satisfy $y_{i} \leq x_{i}$ for $1 \leq i \leq n$. Then using the assumption $F(y) \leq G(y)$ for $y \in S$ we obtain

$$
\begin{aligned}
F(x)= & P\left(X_{1} \leq x_{1}, \ldots, X_{n} \leq x_{n}\right) \\
= & P\left(X_{1} \leq y_{1}, X_{2} \leq x_{2}, \ldots, X_{n} \leq x_{n}\right)+P\left(y_{1}<X_{1} \leq x_{1}, X_{2} \leq x_{2}, \ldots, X_{n} \leq x_{n}\right) \\
= & P\left(X_{1} \leq y_{1}, X_{2} \leq y_{2}, X_{3} \leq x_{3}, \ldots, X_{n} \leq x_{n}\right)+P\left(y_{1}<X_{1} \leq x_{1}, X_{2} \leq x_{2}, \ldots, X_{n} \leq x_{n}\right) \\
& \quad+P\left(X_{1} \leq y_{1}, y_{2}<X_{2} \leq x_{2}, X_{3} \leq x_{3}, \ldots, X_{n} \leq x_{n}\right) \\
= & P\left(X_{1} \leq y_{1}, \ldots, X_{n} \leq y_{n}\right)+P\left(y_{1}<X_{1} \leq x_{1}, X_{2} \leq x_{2}, \ldots, X_{n} \leq x_{n}\right) \\
& \quad+\cdots+P\left(X_{1} \leq y_{1}, \ldots, X_{n-1} \leq y_{n-1}, y_{n}<X_{n} \leq x_{n}\right) \\
& \quad \cdots(y)+\sum_{i=1}^{n}\left(F_{i}\left(x_{i}\right)-F_{i}\left(y_{i}\right)\right) .
\end{aligned}
$$


Considering all $y \in S$ and taking the infimum, this implies by the classical Hoeffding-Fréchet bounds that $F \leq F^{\star}$.

(ii) Similarly to (i), for $y \in S$ satisfying $y_{i} \geq x_{i}$ for $1 \leq i \leq n$ we obtain that

$$
\begin{aligned}
F(x)= & P\left(X_{1} \leq x_{1}, \ldots, X_{n} \leq x_{n}\right) \\
= & P\left(X_{1} \leq y_{1}, \ldots, X_{n} \leq y_{n}\right)-P\left(x_{1}<X_{1} \leq y_{1}, X_{2} \leq x_{2}, \ldots, X_{n} \leq x_{n}\right) \\
& \quad-\cdots-P\left(X_{1} \leq y_{1}, \ldots, X_{n-1} \leq y_{n-1}, x_{n}<X_{n} \leq y_{n}\right) .
\end{aligned}
$$

Considering that $F(y) \geq G(y)$ is equivalent to $1-F(y) \leq 1-G(y)$, it follows that

$$
\begin{aligned}
& 1-F(x)=1- P\left(X_{1} \leq y_{1}, \ldots, X_{n} \leq y_{n}\right)+P\left(x_{1}<X_{1} \leq y_{1}, X_{2} \leq x_{2}, \ldots, X_{n} \leq x_{n}\right) \\
&+\cdots+P\left(X_{1} \leq y_{1}, \ldots, X_{n-1} \leq y_{n-1}, x_{n}<X_{n} \leq y_{n}\right) \\
& \leq 1-G(y)+\sum_{i=1}^{n}\left(F_{i}\left(y_{i}\right)-F_{i}\left(x_{i}\right)\right)
\end{aligned}
$$

which implies

$$
F(x) \geq G(y)-\sum_{i=1}^{n}\left(F_{i}\left(y_{i}\right)-F_{i}\left(x_{i}\right)\right) .
$$

Considering all $y \in S$ and taking the supremum, this implies by the classical Hoeffding-Fréchet bounds that $F \geq F_{\star}$. (iii) is a consequence of (i) and (ii).

\section{Remark 3.}

1) In the case $n=2$ improved Hoeffding-Fréchet bounds have been first derived in [28]. These bounds were restated in the case of uniform marginals, i.e. for copulas, in [34] for the case of equality constraints, where also a sharpness result for increasing sets $S$ and an application to model free pricing bounds for multiasset options is given. In the case $S$ is a singleton and $n=2$ sharpness of this bound is shown in [22], while extensions of the sharpness result are provided in [1]. The paper [2] discuss as application the case where $S$ is the central part of the distribution. We will deal with this case in Section 3.2.

2) During the writing of this paper we became aware that the improved Hoeffding-Fréchet bounds as in Theorem 3.1 were given independently in the recent preprint [16] and used in applications to model-free finance. These authors also show that unlike in the two-dimensional case the improved bounds are copulas only in rather degenerated cases. Theorem 3.1 as stated here however appeared already earlier in the thesis of one of the authors; see [17]. In this context it is also worth mentioning that improved high-dimensional Hoeffding-Fréchet bounds have been also derived in [29] for $S$ being a singleton and in [32] for copulas with known values at several points.

3) If $X$ is positive lower orthant dependent (PLOD), i.e.

$$
F(x) \geq G(x)=\prod_{i=1}^{n} F_{i}\left(x_{i}\right), x \in S=\mathbb{R}^{n},
$$

then

$$
\sup _{y \in \mathbb{R}^{n}}\left\{\prod_{i=1}^{n} F_{i}\left(y_{i}\right)-\sum_{i=1}^{n}\left(F_{i}\left(y_{i}\right)-F_{i}\left(x_{i}\right)\right)_{+}\right\}=\prod_{i=1}^{n} F_{i}\left(x_{i}\right)=G(x)
$$

and as consequence $F_{\star}(x)=\prod_{i=1}^{n} F_{i}\left(x_{i}\right)=G(x)$ coincides with $G$ and is a sharp lower bound. Similarly if $G$ is a joint distribution function with marginals $F_{1}, \ldots, F_{n}$ and $S=\mathbb{R}^{n}$, then the improved HoeffdingFréchet bounds coincide with $G$ and are sharp, i.e. $F^{\star}=G$ under condition (i) and $F_{\star}=G$ under condition (ii).

4) In the particular case where $F_{i}=U[0,1], 1 \leq i \leq n$, Theorem 3.1 implies the following improved bounds for the copula of a risk vector. 
Corollary 3.2 (Improved copula bounds). Let $S \subset[0,1]^{n}$ and let $Q$ be a componentwise increasing function on $[0,1]^{n}$ such that $W(u) \leq Q(u) \leq M(u), u \in[0,1]^{n}$. Define the bounds $A^{S, Q}, B^{S, Q}:[0,1]^{n} \rightarrow[0,1]$ as

$$
\begin{aligned}
& A^{S, Q}(u)=\min \left(M(u), \inf _{a \in S}\left\{Q(a)+\sum_{i=1}^{n}\left(u_{i}-a_{i}\right)_{+}\right\}\right), \\
& B^{S, Q}(u)=\max \left(W(u), \sup _{a \in S}\left\{Q(a)-\sum_{i=1}^{n}\left(a_{i}-u_{i}\right)_{+}\right\}\right) .
\end{aligned}
$$

Then for a n-dimensional copula $C$, it holds that

(i) If $C(u) \leq Q(u)$ for all $u \in S$, then $C(u) \leq A^{S, Q}(u)$, for all $u \in[0,1]^{n}$.

(ii) If $C(u) \geq Q(u)$ for all $y \in S$, then $C(u) \geq B^{S, Q}(u)$, for all $u \in[0,1]^{n}$.

(iii) If $C(u)=Q(u)$ for all $y \in S$, then $B^{S, Q}(u) \leq C(u) \leq A^{S, Q}(u)$, for all $u \in[0,1]^{n}$.

The improved Hoeffding-Fréchet bounds given in Theorem 3.1 may be considerable improvements of the classical Hoeffding-Fréchet bounds in (1.2) and thus may lead to strongly improved VaR bounds for the aggregated risk by the method of improved standard bounds described in Section 2 . The degree of improvement depends on the dependence information described by $S$ and $G$. We analyse some different cases in the applications to follow.

\subsection{Application I: positive dependence in the tails}

We first consider the computation of an upper bound on the $\alpha$-VaR for a risk vector with fixed marginal distributions and positive dependence assumed only in the upper tails of its distribution. Using Theorem 2.3 with Corollary 3.2 we obtain the following result.

Theorem 3.3. Assume that the risk vector $\left(X_{1}, \ldots, X_{n}\right)$ has fixed marginal distributions $F_{1}, \ldots, F_{n}$ and copula $C_{X} \geq C_{l}$ on $S=[\beta, 1]^{n}$, with $\beta \leq \alpha$. Then, we have that

$$
\operatorname{VaR}_{\alpha}(S) \leq \operatorname{VaR}_{\alpha^{\star}}^{+}(S)=\sum_{i=1}^{n} F_{i}^{-1}\left(\alpha^{\star}\right)
$$

where $\alpha^{\star}=\delta_{C_{l}}^{-1}(\alpha)$.

Proof. For a fixed $u \in[\alpha, 1]^{n}$, we have

$$
\max _{a \in[\beta, 1]^{n}}\left\{C_{l}(a)-\sum_{i=1}^{n}\left(a_{i}-u_{i}\right)_{+}\right\}=\max _{a \in[u, 1]^{n}}\left\{C_{l}(a)-\sum_{i=1}^{n}\left(a_{i}-u_{i}\right)\right\}=C_{l}(u) .
$$

The first equality in (3.3) follows by noting that, by componentwise increasingness of the function $C_{l}$, we can always change any $a_{j}<u_{j}$ to $u_{j}$ not decreasing the value of the max in (3.3). Since that the maximum is now taken over $[u, 1]^{n}$, the second inequality follows from the Lipschitz inequality for copulas

$$
C_{l}(a)-C_{l}(u) \leq \sum_{i=1}^{n}\left(a_{i}-u_{i}\right)
$$

see for instance Theorem 1.5.1 in [9]. The theorem then follows from Theorem 2.3 applied with $C_{l}=B^{S, C_{l}}$ as, for $u \geq \alpha$, by (3.3) we have that $\delta_{B^{s, c_{l}}}(u)=\delta_{C_{l}}(u)$. 


\section{Remark 4.}

1) Theorem 3.3 gives the same VaR bound of Theorem 2.3 under the weaker assumption that the positive dependence assumption $C_{X} \geq C_{l}$ only holds in upper $\alpha$-tails of the distribution of $X$, where $\alpha$ is the confidence level at which the VaR is computed. A similar behaviour that an upper VaR bound neglects information on the lower part of a distribution can be also found for the sharp upper VaR bounds without dependence information as given in [24].

2) The computation of $\operatorname{VaR}_{\alpha^{\star}}^{+}(S)$ is straightforward for any number and type of marginal distributions and for any copula lower bound used in banking and insurance practice. This simplicity makes the VaR bound in (3.2) particularly appealing for applications of interest in quantitative risk management.

3) There exist some statistical procedures to test the lower bound on a copula on a subset of its domain. In [33] for instance a Kolmogorov-Smirnov test for quadrant dependence is presented. This can be easily modified to a test for positive quadrant dependence on a subset $S$. In [5] the authors discuss extensively the various statistical methods available to get the ordering information on the distribution. They also discuss methods for the choice of the subset $S$ and also give also a list of relevant references on this subject.

As a first application of Theorem 3.3, we consider a risk portfolio $X$ with identical marginal distributions $F_{1}=$ $\cdots=F_{n}=F$ and portfolio copula $C_{X}$ bounded by a Gumbel copula. Formally, we assume $C_{X} \geq C_{l}=C_{\theta}^{G u}$ on $S=[\alpha, 1]^{n}$. In Table 1 we report the values of $\operatorname{VaR}_{\alpha^{*}}^{+}(S)$ for increasing values of $\theta$, corresponding to increasing dependence assumed in the tails of $X$. The VaR bounds are confronted with sharp upper bounds $\overline{\mathrm{VaR}}_{\alpha}(S)$ obtained without dependence information and the comonotonic value $\operatorname{VaR}_{\alpha}^{+}(S)$. From the table it is clear that one obtains improvements of the sharp bounds for values of $\theta$ large enough. Coherently with the theoretical results presented so far, in the limit $\theta \rightarrow \infty$ we have that $C_{\theta}^{G u} \rightarrow M$ and, consequently, $\operatorname{VaR}_{\alpha^{*}}^{+}(S)$ converges to the comonotonic VaR value $\operatorname{VaR}_{\alpha}^{+}(S)$. We also notice that, according to Theorem 2.2 and Remark 2, in this homogeneous case one gets $\tau_{C_{l}}^{-1}(\alpha)=\operatorname{VaR}_{\alpha^{\star}}^{+}(S)=n F^{-1}\left(\alpha^{\star}\right)$.

Analogous conclusions can be drawn when a Gaussian copula is assumed as a lower bound on the copula of the portfolio, i.e. $C_{X} \geq C_{l}=C_{\rho}^{G a}$ on $S=[\alpha, 1]^{n}$. In Table 2 we collect figures for increasing values of the correlation parameter $\rho$, chosen as to match the corresponding values of the pairwise Kendall's rank correlation of the Gumbel copula in Table 1. Given the same strength of dependence, the Gaussian copula yields a smaller relative improvement with respect to the Gumbel copula. Considering a inhomogeneous portfolio is straightforward as the value of $\alpha^{\star}$ in (3.2) does not depend on the choice of the marginal distributions $F_{i}$. Results for an inhomogeneous portfolio are reported in Table 3.

Table 1 Values for $\operatorname{VaR}_{\alpha^{\star}}^{+}(S)$ (see (3.2)) and $\operatorname{VaR}_{\alpha}^{+}(S)$ (see (1.3)) for a risk vector of $n=5$ risks identically distributed as a Pareto(2), i.e. $F_{i}=F(x)=1-(1+x)^{-2}, x>0,1 \leq i \leq n$, and a Gumbel copula lower bound $C_{X} \geq C_{\theta}^{\mathrm{Gu}}$ on $S=[\alpha, 1]^{n}$. Estimates of $\overline{\mathrm{VaR}}_{\alpha}(S)$ have been computed via Proposition 4 in [14].

\begin{tabular}{ccccccc}
\hline & & \multicolumn{5}{c}{$\operatorname{VaR}_{\alpha^{*}}^{+}(S)$} \\
\cline { 3 - 6 }$n=5$ & $\overline{\operatorname{VaR}}_{\alpha}(S)$ & $\theta=1$ & $\theta=3$ & $\theta=5$ & $\theta=10$ & $\operatorname{VaR}_{\alpha}^{+}(S)$ \\
\hline$\alpha=0.990$ & 84.44 & 107.38 & 60.27 & 53.68 & 49.17 & 45.00 \\
$\alpha=0.995$ & 121.49 & 152.33 & 87.38 & 78.02 & 71.62 & 65.71 \\
$\alpha=0.999$ & 277.84 & 348.20 & 201.72 & 180.71 & 166.36 & 153.11 \\
\hline
\end{tabular}


Table 2 The same as Table 1 with a Gaussian copula lower bound $C_{X} \geq C_{\rho}^{\mathrm{Ga}}$ on $S=[\alpha, 1]^{n}$. The four values chosen for the correlation parameter $\rho$ match the corresponding values of the pairwise Kendall's rank correlation of the Gumbel copula in Table 1.

\begin{tabular}{ccccccc}
\hline & & \multicolumn{5}{c}{$\operatorname{VaR}_{\alpha^{*}}^{+}(S)$} \\
\cline { 3 - 6 }$n=5$ & $\overline{\operatorname{VaR}}_{\alpha}(S)$ & $\rho=0$ & $\rho=0.8660$ & $\rho=0.9511$ & $\rho=0.9877$ & $\operatorname{VaR}_{\alpha}^{+}(S)$ \\
\hline$\alpha=0.990$ & 84.44 & 106.58 & 75.97 & 63.37 & 53.96 & 45.00 \\
$\alpha=0.995$ & 121.49 & 152.96 & 112.41 & 93.89 & 79.15 & 65.71 \\
$\alpha=0.999$ & 277.84 & 348.48 & 269.65 & 222.57 & 187.32 & 153.11 \\
\hline
\end{tabular}

Table 3 The same as Table 1 for a inhomogeneous risk vector of $n=9$ risks with $F_{1}=F_{2}=F_{3}=$ Pareto(2), $F_{4}=$ $F_{5}=F_{6}=\operatorname{LogN}(0.2,1)$ and $F_{7}=F_{8}=F_{9}=\operatorname{Gamma}(3,2)$, and Gumbel copula lower bound $C_{X} \geq C_{\theta}^{\mathrm{Gu}}$ on $S=[\alpha, 1]^{n}$. Estimates of $\overline{\operatorname{VaR}}_{\alpha}(S)$ have been computed via the Rearrangement Algorithm; see [14].

\begin{tabular}{ccccccc}
\hline & & \multicolumn{5}{c}{$\operatorname{VaR}_{\alpha^{*}}^{+}(S)$} \\
\cline { 3 - 6 }$n=9$ & $\overline{\operatorname{VaR}}_{\alpha}(S)$ & $\theta=1$ & $\theta=3$ & $\theta=5$ & $\theta=10$ & $\operatorname{VaR}_{\alpha}^{+}(S)$ \\
\hline$\alpha=0.990$ & 165.29 & 232.76 & 144.77 & 132.06 & 123.24 & 114.96 \\
$\alpha=0.995$ & 206.04 & 289.42 & 180.52 & 164.53 & 153.51 & 143.23 \\
$\alpha=0.999$ & 349.62 & 510.77 & 305.86 & 277.23 & 257.74 & 239.79 \\
\hline
\end{tabular}

\subsection{Application II: known central domain}

We now consider the case in which a copula lower bound is assumed in the central domain of the distribution or any subset $S \subset \mathbb{R}^{n}$ not intersecting the upper $\alpha$-tails, i.e. $\bar{S} \cap[\alpha, 1]^{n}=\emptyset$. This example is motivated by the applications treated in [2] and [5], where for a risk portfolio it is assumed that the distribution is known by statistical analysis in the central domain of the distribution while generally only marginals are known. Within our setting, no improvement is obtained.

Theorem 3.4. Assume that the risk vector $\left(X_{1}, \ldots, X_{n}\right)$ has fixed marginal distributions $F_{1}, \ldots, F_{n}$ and copula $C \geq C_{l}$ on $S \subset \mathbb{R}^{n}$, with $\bar{S} \cap[\alpha, 1]^{n}=\emptyset$. Then, we have that

$$
\operatorname{VaR}_{\alpha}(S) \leq \sum_{i=1}^{n} F_{i}^{-1}\left(\alpha^{*}\right),
$$

where $\alpha^{\star}=\delta_{W}^{-1}(\alpha)$.

Proof. We observe that for any $u \in S$ we have $u_{i}<\alpha$ for at least one coordinate $i$. From $C_{l}(u) \leq M(u)=$ $\min _{1 \leq i \leq n}\left\{u_{i}\right\}<\alpha$, we obtain

$$
\sup _{a \in S}\left\{C_{l}(a)-\sum_{i=1}^{n}\left(a_{i}-u\right)_{+}\right\} \leq \sup _{a \in S}\left\{C_{l}(a)\right\}<\alpha .
$$

As a consequence, the inequality $\delta_{B^{s, c_{l}}}(u) \geq \alpha$ can only be satisfied at those values of $u$ such that

$$
\delta_{B^{s, c_{l}}}(u)=(n u-n+1)_{+}=\delta_{W}(u) .
$$

The theorem then follows from Theorem 2.3 applied with $C_{l}=B^{S, C_{l}}$. 


\section{Remark 5.}

1) Theorem 3.4 implies that under the dependence information on a central part of the distribution, one obtains the standard bound on VaR. For $n>2$, standard bounds are in general improved by sharp upper bounds $\overline{\operatorname{VaR}}_{\alpha}(S)$ obtained without dependence information.

2) From the proof of Theorem 3.4 it follows that in order to obtain the comonotonic bound $\operatorname{VaR}_{\alpha}^{+}(S)$, it is sufficient to assume $C_{X} \geq C_{l}=M$ just at the single point $S=\{(\alpha, \ldots, \alpha)\}$.

Information on a central domain of the distribution becomes relevant in our setting only if it (partially) covers the upper tails of the joint distribution. If $S=[0, \beta]^{n}$, with $\beta$ sufficiently large (i.e. when $\delta_{C_{l}}(\beta) \geq \alpha$ ), then the dependence information covers the relevant part of the distribution tails and the same VaR bounds as in Theorem 3.3 holds. Otherwise, no improvement follows.

Theorem 3.5. Assume that the risk vector $\left(X_{1}, \ldots, X_{n}\right)$ has fixed marginal distributions $F_{1}, \ldots, F_{n}$ and copula $C \geq C_{l}$ on $S=[0, \beta]^{n}$, with $\beta \geq \alpha$. Then:

1) If $\delta_{C_{l}}(\beta) \geq \alpha$, then the VaR bound in Theorem 3.3 holds.

2) If $\delta_{C_{l}}(\beta)<\alpha$, then the VaR bound in Theorem 3.4 holds.

Proof. Assume $S=[0, \beta]^{n}$ with $\beta \geq \alpha$. From the proof of Theorem 3.3 it follows that for any $u \in[0, \beta]^{n}$ we have

$$
\max _{a \in[0, \beta]^{n}}\left\{C_{l}(a)-\sum_{i=1}^{n}\left(a_{i}-u_{i}\right)_{+}\right\}=C_{l}(u) .
$$

If $\delta_{C_{l}}(\beta) \geq \alpha$, then the same VaR bound as in Theorem 3.3 follows analogously.

If instead $\delta_{C_{l}}(\beta)<\alpha$, then we have for all $u$ that

$$
\max _{a \in[0, \beta]^{n}}\left\{C_{l}(a)-\sum_{i=1}^{n}\left(a_{i}-u\right)_{+}\right\} \leq \max _{a \in[0, \beta]^{n}}\left\{C_{l}(a)\right\} \leq C_{l}(\beta, \ldots, \beta)<\alpha
$$

and the inequality $\delta_{B^{s, c_{l}}}\left(\alpha^{\star}\right) \geq \alpha$ can only be satisfied for those values of $u$ such that

$$
\delta_{B^{s, c_{l}}}(u)=(n u-n+1)_{+}=\delta_{W}(u) ;
$$

and the same conclusion as in Theorem 3.4 follows.

Tables 4-5 show the values of $\alpha^{\star}$, depending on the fixed quantile level $\alpha$ and on the copula $C_{l}$. They can be interpreted as the minimal value of $\beta$ at which dependence information put on $S=[0, \beta]^{n}$ becomes significant.

\subsection{Application III: positively dependent subgroups with an additional internal tail dependence assumption}

As a final application we modify the model assumption investigated in [6], where the authors consider the case that the $n$ risks of the risk vector $X$ are split into $k$ positively dependent subgroups $I_{i}$ of cardinality $n_{i}=$ $\left|I_{i}\right|$, with $\sum_{i=1}^{k} n_{i}=n$. The authors of [6] allow any kind of dependence within these subgroups. Within our framework, we assume that the copula $C_{X}^{i}$ of the subvector $\left(X_{j}, j \in I_{i}\right)$ satisfies $C_{X}^{i} \geq C_{l}^{i}$ on $S=[\alpha, 1]^{n_{i}}$. This implies the risk portfolio lower bound $C_{X} \geq C_{l}$ on $S=[\alpha, 1]^{n}$ with

$$
C_{l}\left(u_{1}, \ldots, u_{n}\right)=\prod_{i=1}^{k} C_{l}^{i}\left(u_{j}, j \in I_{i}\right) .
$$


Table 4 Values for $\alpha^{\star}$ for a $n$-dimensional Gumbel copula $C_{\theta}^{G u}$.

\begin{tabular}{|c|c|c|c|c|c|c|c|}
\hline$n=5$ & & & & $n=9$ & & & \\
\hline$\alpha$ & $\theta=3$ & $\theta=5$ & $\theta=10$ & $\alpha$ & $\theta=3$ & $\theta=5$ & $\theta=10$ \\
\hline 0.990 & 0.99413 & 0.99274 & 0.99148 & 0.990 & 0.99517 & 0.99354 & 0.99196 \\
\hline 0.995 & 0.99707 & 0.99637 & 0.99574 & 0.995 & 0.99759 & 0.99677 & 0.99598 \\
\hline 0.999 & 0.99942 & 0.99928 & 0.99915 & 0.999 & 0.99952 & 0.99936 & 0.99920 \\
\hline
\end{tabular}

Table 5 Values for $\alpha^{\star}$ for a $n$-dimensional Gaussian copula $C_{\rho}^{G a}$.

\begin{tabular}{|c|c|c|c|c|c|c|c|}
\hline$n=5$ & & & & $n=9$ & & & \\
\hline$\alpha$ & $\rho=0.8660$ & $\rho=0.9511$ & $\rho=0.9877$ & $\alpha$ & $\rho=0.8660$ & $\rho=.9511$ & $\rho=0.9877$ \\
\hline 0.990 & 0.99618 & 0.99465 & 0.99282 & 0.990 & 0.99720 & 0.99559 & 0.99346 \\
\hline 0.995 & 0.99819 & 0.99744 & 0.99647 & 0.995 & 0.99869 & 0.99791 & 0.99683 \\
\hline 0.999 & 0.99966 & 0.99952 & 0.99934 & 0.999 & 0.99977 & 0.99963 & 0.99940 \\
\hline
\end{tabular}

Table 6 Values for $\operatorname{VaR}_{\alpha^{\star}}^{+}(S)$ (see (3.2)) for a risk vector of $n=8$ risks identically distributed as a Pareto(2) under the framework described in Section 3.3.

\begin{tabular}{llcccc}
\hline & & \multicolumn{4}{c}{$\operatorname{VaR}_{\alpha^{*}}^{+}(S)$} \\
\cline { 3 - 6 }$\theta=3$ & $\overline{\operatorname{VaR}}_{\alpha}(S)$ & $k=1$ & $k=2$ & $k=4$ & $k=8$ \\
\hline$\alpha=0.990$ & 141.67 & 104.85 & 133.77 & 172.53 & 219.79 \\
$\alpha=0.995$ & 203.66 & 151.80 & 193.04 & 244.7 & 309.22 \\
$\alpha=0.999$ & 465.29 & 349.68 & 442.52 & 559.35 & 706.29 \\
\hline
\end{tabular}

\begin{tabular}{llcccc} 
& & \multicolumn{5}{c}{$\operatorname{VaR}_{\alpha^{*}}^{+}(S)$} \\
\cline { 3 - 6 }$\theta=5$ & $\overline{\operatorname{VaR}}_{\alpha}(S)$ & $k=1$ & $k=2$ & $k=4$ & $k=8$ \\
\hline$\alpha=0.990$ & 141.67 & 90.364 & 121.43 & 164.34 & 219.79 \\
$\alpha=0.995$ & 203.66 & 131.20 & 175.42 & 233.43 & 309.22 \\
$\alpha=0.999$ & 465.29 & 303.42 & 402.80 & 533.79 & 706.29 \\
\hline
\end{tabular}

\begin{tabular}{llcccc} 
& & \multicolumn{4}{c}{$\operatorname{VaR}_{\alpha^{*}}^{+}(S)$} \\
\cline { 3 - 6 }$\theta=10$ & $\overline{\operatorname{VaR}}_{\alpha}(S)$ & $k=1$ & $k=2$ & $k=4$ & $k=8$ \\
\hline$\alpha=0.990$ & 141.67 & 80.71 & 112.86 & 156.29 & 219.79 \\
$\alpha=0.995$ & 203.66 & 117.50 & 163.21 & 225.29 & 309.22 \\
$\alpha=0.999$ & 465.29 & 272.69 & 375.32 & 515.38 & 706.29 \\
\hline
\end{tabular}

As a concrete example we consider an homogeneous portfolio with $n=8$ Pareto(2) risks with $k$ subgroups of equal size $n_{i}=n / k$ and copula lower bounds $C_{l}^{i}=C_{\theta}^{\mathrm{Gu}}$. Table 6 summarizes the figures of the VaR bound $\operatorname{VaR}_{\alpha^{*}}^{+}(S)$ given in Theorem 3.3. From Table 6 one can see a considerable reduction for $k=1,2$. The case $k=n$ represents the case in which the risk portfolio is assumed to be PLOD, and one cannot expect a reduction here; see also Section 3 in [14]. In the limit as $\theta \rightarrow \infty$ one gets the figures reported in Table 4 in [6]. 


\section{Concluding remarks}

This paper contributes to the streamline of easily computable and practical bounds on the VaR of a joint portfolio of risks. Based on a novel extension of classical Hoeffding-Fréchet bounds, we provide an upper VaR bound for a joint risk portfolio with fixed marginal distributions and positive dependence information assumed on a subset of the domain of its distribution function. The newly provided VaR bound can be interpreted as a comonotonic VaR computed at a distorted confidence level and its quality is illustrated in a series of examples.

In all the examples considered we obtain a remarkable reduction of the VaR bound when moderate or strong positive dependence in the upper tails of the joint distribution is assumed. On the other hand, when dependence information is known only on the central part of the distribution we do not get any improvement.

In general the problem of how to find sharp (best-possible) bounds on the VaR of a joint risk portfolio when positive dependence information of the type $C_{X} \geq C_{l}$ is known on the portfolio copula $C_{X}$ remains open.

Acknowledgement: The authors thank the guest editor Steven Vanduffel and two anonymous referees for their careful reading of the paper and the many suggestions given on an earlier version.

\section{References}

[1] Bernard, C., X. Jiang, and S. Vanduffel (2012). A note on 'Improved Fréchet bounds and model-free pricing of multi-asset options' by Tankov (2011). J. Appl. Probab. 49(3), 866-875.

[2] Bernard, C., Y. Liu, N. MacGillivray, and J. Zhang (2013). Bounds on capital requirements for bivariate risk with given marginals and partial information on the dependence. Depend. Model. 1, 37-53.

[3] Bernard, C., L. Rüschendorf, and S. Vanduffel (2014). Value-at-risk bounds with variance constraints. J. Risk Insur., in press. DOI: $10.1111 /$ jori.12108.

[4] Bernard, C., L. Rüschendorf, S. Vanduffel, and J. Yao (2015). How robust is the Value-at-Risk of credit risk portfolios? Eur. J. Finance, in press. DOI: 10.1080/1351847X.2015.1104370.

[5] Bernard, C. and S. Vanduffel (2015). A new approach to assessing model risk in high dimensions. J. Bank. Financ. 58, 166-178.

[6] Bignozzi, V., G. Puccetti, and L. Rüschendorf (2015). Reducing model risk via positive and negative dependence assumptions. Insurance Math. Econom. 61, 17-26.

[7] Dall'Aglio, G. (1972). Fréchet classes and compatibility of distribution functions. Symp. Math. 9, 131-150.

[8] Dolati, A. and A. Dehgan Nezhad (2014). Some results on convexity and concavity of multivariate copulas. Iran. J. Math. Sci. Inform. 9(2), 87-100, 129.

[9] Durante, F. and C. Sempi (2016). Principles of Copula Theory. CRC Press, Boca Raton FL.

[10] Embrechts, P., A. Höing, and A. Juri (2003). Using copulae to bound the Value-at-Risk for functions of dependent risks. Finance Stoch. 7(2), 145-167.

[11] Embrechts, P. and G. Puccetti (2006a). Aggregating risk capital, with an application to operational risk. Geneva Risk Insur. Rev. 31(2), 71-90.

[12] Embrechts, P. and G. Puccetti (2006b). Bounds for functions of dependent risks. Finance Stoch. 10(3), 341-352.

[13] Embrechts, P. and G. Puccetti (2010). Bounds for the sum of dependent risks having overlapping marginals. J. Multivariate Anal. 101(1), 177-190.

[14] Embrechts, P., G. Puccetti, and L. Rüschendorf (2013). Model uncertainty and VaR aggregation. J. Bank. Financ. 58(8), 2750-2764.

[15] Embrechts, P., G. Puccetti, L. Rüschendorf, R. Wang, and A. Beleraj (2014). An academic response to Basel 3.5. Risks 2(1), 25-48.

[16] Lux, T. and A. Papapantoleon (2016). Improved Fréchet-Hoeffding bounds on $d$-copulas and applications in model-free finance. Preprint, available at http://arxiv.org/abs/1602.08894.

[17] Manko, D. (2015). Verteilungsschranken für Portfolios von Abhängigen Risiken. Masterthesis University Freiburg.

[18] Marshall, A. W., I. Olkin, and B. C. Arnold (2011). Inequalities: Theory of Majorization and its Applications. Second edition. Springer, New York.

[19] McNeil, A. J., R. Frey, and P. Embrechts (2015). Quantitative Risk Management: Concepts, Techniques, Tools. Revised Editon. Princeton University Press, Princeton. 
[20] Mesfioui, M. and J.-F. Quessy (2005). Bounds on the value-at-risk for the sum of possibly dependent risks. Insurance Math. Econom. 37(1), 135-151.

[21] Nelsen, R. B. (2006). An Introduction to Copulas. Second Edition. Springer, New York.

[22] Nelsen, R. B., J. J. Quesada Molina, J. A. Rodríguez Lallena, and M. Úbeda Flores (2004). Best-possible bounds on sets of bivariate distribution functions. J. Multivariate Anal. 90(2), 348-358.

[23] Puccetti, G. and L. Rüschendorf (2012a). Bounds for joint portfolios of dependent risks. Stat. Risk Model. 29(2), $107-131$.

[24] Puccetti, G. and L. Rüschendorf (2012b). Computation of sharp bounds on the distribution of a function of dependent risks. J. Comput. Appl. Math. 236(7), 1833-1840.

[25] Puccetti, G. and L. Rüschendorf (2013). Sharp bounds for sums of dependent risks. J. Appl. Probab. 50(1), 42-53.

[26] Puccetti, G., L. Rüschendorf, D. Small, and S. Vanduffel (2015). Reduction of Value-at-Risk bounds via independence and variance information. Scand. Actuar. J., in press. DOI: 10.1080/03461238.2015.1119717.

[27] Puccetti, G. and R. Wang (2015). Extremal dependence concepts. Stat. Science 30(4), 485-517.

[28] Rachev, S. T. and L. Rüschendorf (1994). Solution of some transportation problems with relaxed or additional constraints. SIAM J. Control Optim. 32(3), 673-689.

[29] Rodríguez-Lallena, J. A. and M. Úbeda-Flores (2004). Best-possible bounds on sets of multivariate distribution functions. Comm. Statist. Theory Methods 33(4), 805-820.

[30] Rüschendorf, L. (2005). Stochastic ordering of risks, influence of dependence and a. s. construction. In N. Balakrishnan, I. G. Bairamov, and O. L. Gebizlioglu (Eds.), Advances on Models, Characterization and Applications., pp. 19-56. Chapman \& Hall/CRC, Boca Raton FL.

[31] Rüschendorf, L. (2013). Mathematical Risk Analysis. Dependence, Risk Bounds, Optimal Allocations and Portfolios. Springer, Heidelberg.

[32] Sadooghi-Alvandi, S. M., Z. Shishebor, and H. A. Mardani-Fard (2013). Sharp bounds on a class of copulas with known values at several points. Comm. Statist. Theory Methods 42(12), 2215-2228.

[33] Scaillet, O. (2005). A Kolmogorov-Smirnov type test for positive quadrant dependence. Canad. J. Statist. 33(3), $415-427$.

[34] Tankov, P. (2011). Improved Fréchet bounds and model-free pricing of multi-asset options. J. Appl. Probab. 48(2), 389-403.

[35] Wang, R., L. Peng, and J. Yang (2013). Bounds for the sum of dependent risks and worst value-at-risk with monotone marginal densities. Finance Stoch. 17(2), 395-417. 